Article

\title{
An Inverse Source Problem for the Generalized Subdiffusion Equation with Nonclassical Boundary Conditions
}

\author{
Emilia Bazhlekova
}

check for

updates

Citation: Bazhlekova, E. An Inverse Source Problem for the Generalized Subdiffusion Equation with Nonclassical Boundary Conditions. Fractal Fract. 2021, 5, 63. https:// doi.org/10.3390/ fractalfract5030063

Academic Editor: Trifce Sandev

Received: 30 April 2021

Accepted: 26 June 2021

Published: 30 June 2021

Publisher's Note: MDPI stays neutral with regard to jurisdictional claims in published maps and institutional affiliations.

Copyright: (C) 2021 by the author. Licensee MDPI, Basel, Switzerland. This article is an open access article distributed under the terms and conditions of the Creative Commons Attribution (CC BY) license (https:/ / creativecommons.org/licenses/by/ $4.0 /)$.
Institute of Mathematics and Informatics, Bulgarian Academy of Sciences, Acad. G. Bonchev Str., Bl. 8, 1113 Sofia, Bulgaria; e.bazhlekova@math.bas.bg

\begin{abstract}
An initial-boundary-value problem is considered for the one-dimensional diffusion equation with a general convolutional derivative in time and nonclassical boundary conditions. We are concerned with the inverse source problem of recovery of a space-dependent source term from given final time data. Generalized eigenfunction expansions are used with respect to a biorthogonal pair of bases. Existence, uniqueness and stability estimates in Sobolev spaces are established.
\end{abstract}

Keywords: subdiffusion equation; inverse source problem; Riesz basis; Mittag-Leffler function; Stieltjes function

\section{Introduction}

Anomalous (non-Fickian) diffusion processes are modeled by employing different types of fractional partial differential and integro-differential equations [1]. Analytical and numerical solutions to fractional differential equations have been investigated by several authors, see e.g., [2,3]. Recently, generalized subdiffusion equations with different memory kernels are extensively studied as models that unify a wide range of anomalous diffusion patterns [4-6].

In this work we consider the generalized subdiffusion equation in the form

$$
\mathbb{D}_{t}^{(k)} u(x, t)=u_{x x}(x, t)+F(x, t),
$$

where $\mathbb{D}_{t}^{(k)}$ is an integro-differential operator defined by

$$
\left(\mathbb{D}_{t}^{(k)} f\right)(t)=\frac{\mathrm{d}}{\mathrm{d} t} \int_{0}^{t} k(t-\tau)(f(\tau)-f(0)) \mathrm{d} \tau, t>0
$$

with a locally integrable memory kernel $k(t)$. For the kernel $k(t)$ we also assume that its Laplace transform $\widehat{k}(s)$ exists for all $s>0$ and

$$
\widehat{k}(s) \in \mathcal{S F}, \lim _{s \rightarrow+\infty} s \widehat{k}(s)=+\infty
$$

where $\mathcal{S F}$ denotes the class of Stieltjes functions (the definition of this class is given in the next section). The most prominent particular examples of operators $\mathbb{D}_{t}^{(k)}$ are the first-order derivative $\frac{\mathrm{d}}{\mathrm{d} t}$, corresponding to $\widehat{k}(s)=1$, the Caputo time-fractional derivative of order $\alpha \in(0,1)$, where $\widehat{k}(s)=s^{\alpha-1}$, as well as linear combinations with positive coefficients of such derivatives.

Let us note that assumptions (3) are weaker than those required in the definition of the so-called general fractional derivative, introduced in [7] and studied in detail in [8,9]. The operator $\mathbb{D}_{t}^{(k)}$ is a general fractional derivative, if, along with (3), the following additional limiting behavior conditions are imposed: $\widehat{k}(s) \rightarrow 0$ as $s \rightarrow \infty ; \widehat{k}(s) \rightarrow \infty$ and $s \widehat{k}(s) \rightarrow 0$ as $s \rightarrow 0$. To cover some examples of physically meaningful models with corresponding 
memory kernels, which do not satisfy some of the additional conditions, they are not required in this work. Such are, for instance, the subdiffusion equation with the truncated power-law memory kernel $k(t)=e^{-\gamma t} \frac{t^{-\alpha}}{\Gamma(1-\alpha)}, \gamma>0, \alpha \in(0,1)$, considered in [4,6], the fractal mobile/immobile solute transport equation introduced in [10], and the Jeffreys' type heat conduction model in the diffusion regime [11,12]. On the other hand, the assumption $\widehat{k}(s) \in \mathcal{S F}$ is typical for a subdiffusion model (see e.g., [4-6]) and allows the use of the convenient Bernstein functions technique [13]. It implies that the kernel $k(t)$ admits the representation

$$
k(t)=k_{0} \delta(t)+k_{1}(t),
$$

where $k_{0} \geq 0, \delta(\cdot)$ denotes the Dirac delta function, and $k_{1}(t) \in L_{\text {loc }}^{1}\left(\mathbb{R}_{+}\right)$is a completely monotone function, i.e. it is of class $C^{\infty}\left(\mathbb{R}_{+}\right)$and

$$
(-1)^{n} k_{1}^{(n)}(t) \geq 0, \quad t>0, n=0,1,2, \ldots
$$

For example, in the case of the first-order derivative $k_{0}=1$ and $k_{1} \equiv 0$, while $k_{0}=0$ and $k_{1}=\frac{t^{-\alpha}}{\Gamma(1-\alpha)}$ for the Caputo time-fractional derivative of order $\alpha \in(0,1)$.

A more general setting for the subdiffusion equation (1) has been introduced in [14] and further developed and applied in e.g., $[15,16]$. In this setting, it is assumed that the function $k_{1}(t)$ in representation (4) is non-negative and non-increasing (satisfies (5) only for $n=0,1)$ and there exists a locally integrable kernel $l(t)$, such that $(k * l)(t)=1, t>0$. The kernel $l(t)$ with these properties is referred to as completely positive kernel. Let us note that assumptions (3) also ensure the existence of such kernel $l(t)$, which in this case is completely monotone.

In the present work we are concerned with an inverse source problem for the subdiffusion Equation (1). Different kinds of inverse problems for the diffusion equation with the Caputo time-derivative of order $\alpha \in(0,1)$ are extensively studied recently, see e.g., [17-21]. For a comprehensive tutorial on inverse problems for anomalous diffusion processes we refer to [22]. Identification of a space-dependent source factor $h(x)$ in a source function of the form $F(x, t)=h(x) q(x, t)$ from final overdetermination are studied in [17,19,23-25], where different assumptions on the known source factor $q(x, t)$ are discussed. Concerning the generalized subdiffusion equation, various types of inverse problems for such equations are studied in [26-28].

In this work, we consider the problem of identifying a space-dependent source factor $h(x)$ and the solution $u(x, t)$ to the following nonlocal boundary-value problem with final overdetermination

$$
\begin{aligned}
& \mathbb{D}_{t}^{(k)} u(x, t)=u_{x x}(x, t)+q(t) h(x), \quad x \in(0,1), \quad t \in(0, T), \\
& u(1, t)=0, \quad u_{x}(0, t)=u_{x}(1, t), \quad t \in(0, T], \\
& u(x, 0)=0, \quad u(x, T)=g(x), \quad x \in[0,1],
\end{aligned}
$$

where the operator $\mathbb{D}_{t}^{(k)}$ acting with respect to the time variable is defined in (2), $q(t)$ is a prescribed continuous function, $g(x)$ is a known square integrable function, and $T>0$ is the final time.

In practical applications, the input data $g(x)$ is given by measurement and actually the measured data $g^{v}(x)$ is available, which is merely in $L^{2}(0,1)$ and satisfies

$$
\left\|g^{v}-g\right\|_{L^{2}(0,1)} \leq v,
$$

where the constant $v$ represents the noise level.

The study of nonlocal boundary-value problems is motivated by the fact that in many cases a nonlocal condition is more realistic in treating physical problems than the classical local conditions. Inverse source problems with nonlocal boundary conditions are studied e.g., in [29-34]. The papers $[29,30]$ are concerned with two particular cases of the inverse 
source problem (6)-(8). In these works, existence of a unique solution in the classical sense is established for $\mathbb{D}_{t}^{(k)}=\frac{\mathrm{d}}{\mathrm{d} t}$ and $\mathbb{D}_{t}^{(k)}$-the Caputo time-fractional derivative of order $\alpha \in(0,1)$, respectively, and $q(t)=1$.

In the case of inverse source problem with final overdetermination for the timefractional subdiffusion equation on $\Omega \times(0, T)$ with the classical Dirichlet boundary conditions the following estimates are satisfied (see e.g., $[19,22,35]$ )

$$
C_{1}\|g\|_{H^{2}(\Omega)} \leq\|h\|_{L^{2}(\Omega)} \leq C_{2}\|g\|_{H^{2}(\Omega)} .
$$

The same behavior is observed as well with the classical diffusion equation. Therefore, such inverse problems are moderately ill-posed: the overdetermination function $g(x)$ has a better regularity than the source term $h(x)$, as the regularity loss is two spatial derivatives.

The main goal of the present work is to study stability of the inverse source problem (6)-(8) in Sobolev spaces, and to prove analogous estimates for the overdetermination function $g(x)$ and the source factor $h(x)$. To this end generalized eigenfunction expansions are used with respect to a biorthogonal pair of bases. The present paper is a continuation of the recent work [36], in which inverse source problem (6)-(8) is solved in the classical sense, together with detailed study of particular cases for the operator $\mathbb{D}_{t}^{(k)}$ and some numerical examples.

The rest of the paper is organized as follows. In Section 2 the assumptions on the memory kernel are discussed by the use of the Bernstein functions technique. In Section 3 two examples of generalized subdiffusion equations are considered. Properties of the solution of the generalized relaxation equation are summarized in Section 4. Section 5 is concerned a biorthonormal pair of Riesz bases for the considered problem. In Section 6 formal spectral expansions for the solution are derived. In Section 7 we establish uniqueness and stability estimates for the inverse problem. Concluding remarks are given in Section 8 .

\section{Assumptions on the Memory Kernel}

We start with some preliminaries on completely monotone functions, Bernstein, complete Bernstein and Stieltjes functions. Let us denote the Laplace transform of a function by

$$
\mathcal{L}\{f(t)\}(s)=\widehat{f}(s)=\int_{0}^{\infty} e^{-s t} f(t) \mathrm{d} t .
$$

The class of completely monotone functions, defined in (5), is denoted by $\mathcal{C M F}$. The characterization of the class $\mathcal{C M F}$ is given by the Bernstein's theorem stating that a function is completely monotone if and only if it can be represented as the Laplace transform of a non-negative measure (non-negative function or generalized function).

The class of Stieltjes functions $(\mathcal{S F})$ consists of all functions defined on $\mathbb{R}_{+}$which can be written as a restriction of the Laplace transform of a completely monotone function to the real positive semi-axis. More precisely, $\varphi \in \mathcal{S F}$ if and only if it admits the representation [7]

$$
\varphi(s)=\varphi_{0}+\int_{0}^{\infty} e^{-s t} \psi(t) \mathrm{d} t, \quad s>0,
$$

where $\varphi_{0} \geq 0, \psi \in \mathcal{C M F}$ and the Laplace transform of $\psi$ exists for any $s>0$. Moreover, $\varphi_{0}=\lim _{s \rightarrow+\infty} \varphi(s)$, see e.g., [37], Theorem 2.6.

A non-negative function $\varphi$ on $\mathbb{R}_{+}$is said to be a Bernstein function $(\varphi \in \mathcal{B F})$ if $\varphi^{\prime}(s) \in \mathcal{C M F} ; \varphi(s)$ is said to be a complete Bernstein function $(\mathcal{C B F})$ if and only if

$$
\varphi(s) / s \in \mathcal{S F}, \quad s>0 .
$$

The inclusions $\mathcal{S F} \subset \mathcal{C} \mathcal{M F}$ and $\mathcal{C B F} \subset \mathcal{B F}$ are valid. An example of a completely monotone function is the Mittag-Leffler function $E_{\alpha, \beta}(-s)$ (for definition see (16)) provided $0<\alpha \leq 1$ and $\alpha \leq \beta$. If $\alpha \in[0,1]$ then $s^{\alpha} \in \mathcal{C B F}$ and $s^{\alpha-1} \in \mathcal{S F}$. 
A selection of properties of the classes of functions defined above is given next. For proofs and more details on these special classes of functions we refer to [13,15], Chapter 4.

(P1) The class $\mathcal{C} \mathcal{M F}$ is closed under point-wise multiplication.

(P2) If $\varphi \in \mathcal{C M F}$ and $\psi \in \mathcal{B F}$ then the composite function $\varphi(\psi) \in \mathcal{C M} \mathcal{F}$.

(P3) $\varphi \in \mathcal{C B F}$ if and only if $1 / \varphi \in \mathcal{S F}$.

(P4) If $\varphi \in \mathcal{B F}$ then it admits a continuous extension to $\overline{\mathbb{C}}_{+}$, which is holomorphic in $\mathbb{C}_{+}$ and satisfies $\Re \varphi(s)>0$ for all $\Re s>0$.

We proceed with a short discussion on the assumptions (3) for the kernel $k(t)$ and their implications.

Let us first note that the assumption $\widehat{k}(s) \in \mathcal{S} \mathcal{F}$ implies the non-negativity of the Green function $\mathcal{G}(x, t)$ to Equation (1), which is a necessary condition for a diffusion model. Indeed, in Laplace domain the Green function obeys the identity

$$
\widehat{\mathcal{G}}(x, s)=\frac{\widehat{k}(s)}{2} \exp (-|x| s \widehat{k}(s)), x \in \mathbb{R}, s>0 .
$$

By the Bernstein's theorem, it is sufficient to prove that $\widehat{\mathcal{G}}(x, s) \in \mathcal{C M F}$ for $s>0$ and $|x|$ considered to be a parameter. According to (11), $\widehat{k}(s) \in \mathcal{S F}$ is equivalent to $s \widehat{k}(s) \in \mathcal{C B F}$. Then (P2) implies that $\exp (-|x| s \widehat{k}(s)) \in \mathcal{C} \mathcal{M F}$ as a composition of the completely monotone in $t$ function $e^{-|x| t}$ and the Bernstein function $s \widehat{k}(s)$. Moreover, $\widehat{k}(s) \in \mathcal{S F} \subset \mathcal{C} \mathcal{M} \mathcal{F}$. Therefore, $\widehat{\mathcal{G}}(x, s) \in \mathcal{C} \mathcal{M F}$ as a product of two completely monotone functions, see (P1).

Concerning the limiting behavior of the kernel $k(t)$ the initial value theorem for the Laplace transform implies $\lim _{t \rightarrow 0^{+}} k(t)=\lim _{s \rightarrow \infty} s \widehat{k}(s)=+\infty$. Thus, we restrict our attention to kernels, singular at the origin.

The representation (4) for the kernel $k(t)$ follows from the assumption $\widehat{k}(s) \in \mathcal{S F}$ and the characterization (10) of Stieltjes functions.

Furthermore, we are looking for a corresponding Sonine kernel $l(t)$ to the kernel $k(t)$. This is a function satisfying $l(t) \in L_{l o c}^{1}\left(\mathbb{R}_{+}\right)$, such that

$$
(k * l)(t) \equiv 1
$$

Here $*$ denotes the convolution $(k * l)(t)=\int_{0}^{t} k(t-\tau) l(\tau) \mathrm{d} \tau$. Relation (12) is equivalent to $\widehat{k}(s) \widehat{l}(s)=1 / s$ and by the use of (P3) assumptions (3) imply $\widehat{l}(s) \in \mathcal{S F}$ and $\lim _{s \rightarrow+\infty} \widehat{l}(s)=0$. Therefore, according to (10), under the assumptions (3) a resolvent kernel $l(t)$ exists and $l(t) \in \mathcal{C} \mathcal{M F}$.

Let us note that if $f^{\prime}$ is integrable then $(k * f)^{\prime}(t)=\left(k * f^{\prime}\right)(t)+k(t) f(0)$. Therefore, in this case $\left(\mathbb{D}_{t}^{(k)} f\right)(t)=\left(k * f^{\prime}\right)(t)$ and (12) implies that $\left(\mathbb{D}_{t}^{(k)} f\right)(t)=g(t)$ is equivalent to $f^{\prime}(t)=(l * g)^{\prime}(t)$. This enables us to rewrite Equation (1) as a generalized diffusion equation in the so-called modified form, see e.g., [4]. In [38] we presented arguments why this form is more natural when considering multi-component systems, in particular, systems with a source. Assume that there is a source term $h(x)$ in the conservation law, which depends only on the space variable. Then the subdiffusion equation in modified form reads

$$
u_{t}(x, t)=\frac{\partial}{\partial t}\left(l * u_{x x}\right)(x, t)+h(x)
$$

Applying the convolution operator $(k *)$ to both sides of (13) we obtain

$$
k * u_{t}=k * \frac{\partial}{\partial t}\left(l * u_{x x}\right)+(k * 1)(t) h(x)=\frac{\partial}{\partial t}\left(k * l * u_{x x}\right)-k(t)\left(l * u_{x x}\right)(x, 0)+(k * 1)(t) h(x),
$$

which implies by the use of (12)

$$
\mathbb{D}_{t}^{(k)} u=u_{x x}(x, t)+q(t) h(x),
$$


where $q(t)=\int_{0}^{t} k(\tau) \mathrm{d} \tau$. Therefore, $q(t)$ is a Bernstein function with $q(0)=k_{0} \geq 0$. In particular, it is continuous, non-negative and non-decreasing function.

\section{Two Examples of Generalized Subdiffusion Equations}

In [36] we considered basic examples of kernels $k(t)$, satisfying (3), which do not contain a Dirac delta function in their definition, i.e., $k_{0}=0$ in (4). In this section, two additional physically meaningful examples are given, such that $k_{0}>0$.

The two models are defined in terms of derivatives of fractional order and kernels of Mittag-Leffler type.

The Riemann-Liouville and the Caputo fractional derivatives of order $\alpha \in(0,1)$, denoted by $D_{t}^{\alpha}$ and ${ }^{C} D_{t}^{\alpha}$, respectively, are defined by the identities [39]

$$
\left(D_{t}^{\alpha} f\right)(t)=\frac{\mathrm{d}}{\mathrm{d} t}\left(J_{t}^{1-\alpha} f\right)(t), \quad\left({ }^{C} D_{t}^{\alpha} f\right)(t)=\frac{\mathrm{d}}{\mathrm{d} t}\left(J_{t}^{1-\alpha}(f(\cdot)-f(0))\right)(t), \quad 0<\alpha<1,
$$

where $J_{t}^{\beta}$ denotes the Riemann-Liouville fractional integral:

$$
\left(J_{t}^{\beta} f\right)(t)=\frac{1}{\Gamma(\beta)} \int_{0}^{t}(t-\tau)^{\beta-1} f(\tau) \mathrm{d} \tau, \beta>0 ; \quad J_{t}^{0}=I .
$$

The Mittag-Leffler function is defined by the series [39]

$$
E_{\alpha, \beta}(z)=\sum_{k=0}^{\infty} \frac{z^{k}}{\Gamma(\alpha k+\beta)}, \quad \alpha, \beta, z \in \mathbb{C}, \Re \alpha>0 ; E_{\alpha}(z)=E_{\alpha, 1}(z) ;
$$

and satisfies the Laplace transform pair

$$
\mathcal{L}\left\{t^{\beta-1} E_{\alpha, \beta}\left(-\lambda t^{\alpha}\right)\right\}(s)=\frac{s^{\alpha-\beta}}{s^{\alpha}+\lambda} .
$$

\subsection{Two Time-Scale Diffusion Model}

The time-fractional diffusion equation with the fractional Caputo time-derivative of order $\alpha \in(0,1)$ accurately describes the power-law decaying behavior of the subdiffusive transport of solutes in heterogeneous media. However, the first-order time derivative of the solution to this equation exhibits a nonphysical singularity of order $O\left(t^{\alpha-1}\right)$ near the initial time $t=0$. This disadvantage can be avoided if the following two time-scale model is considered

$$
u_{t}(x, t)+a^{C} D_{t}^{\alpha} u(x, t)=u_{x x}(x, t)+F(x, t), \quad \alpha \in(0,1) .
$$

Equation (18) is introduced in [10] as a model for anomalously diffusive transport of solute in heterogeneous porous media, where it is referred to as Fractal mobile/immobile solute transport equation.

In the case of Equation (18) the corresponding Sonine kernels $k(t)$ and $l(t)$ satisfy the identities

$$
\begin{aligned}
& k(t)=\delta(t)+a \frac{t^{-\alpha}}{\Gamma(1-\alpha)}, \widehat{k}(s)=1+a s^{\alpha-1}, \\
& l(t)=E_{1-\alpha}\left(-a t^{1-\alpha}\right), \quad \widehat{l}(s)=\frac{s^{-\alpha}}{s^{1-\alpha}+a} .
\end{aligned}
$$

\subsection{Fractional Jeffreys-Type Heat Conduction Equation}

According to the fractional Jeffreys-type constitutive law (see [11] Chapter 7) the heat flux $q(x, t)$ and the temperature $u(x, t)$ are related via the equation

$$
\left(1+a D_{t}^{\alpha}\right) q(x, t)=-k\left(1+b D_{t}^{\alpha}\right) u_{x}(x, t),
$$


where $D_{t}^{\alpha}$ denotes the Riemann-Liouville fractional time-derivative of order $\alpha \in(0,1]$, $a>0$ and $b>0$ are generalized relaxation times, $k$ is the thermal conductivity. Combining Equation (19) with the energy balance equation

$$
-\frac{\partial}{\partial x} q(x, t)+J(x, t)=C u_{t}(x, t),
$$

where $C$ denotes the heat capacity and $J(x, t)$ is a heat source, one finds the following fractional Jeffreys-type heat conduction equation

$$
\left(1+a D_{t}^{\alpha}\right) u_{t}(x, t)=\mathcal{D}\left(1+b D_{t}^{\alpha}\right) u_{x x}(x, t)+C^{-1}\left(1+a D_{t}^{\alpha}\right) J(x, t),
$$

where $\mathcal{D}=k / C$ is the thermal diffusivity. For notational simplicity we set $\mathcal{D}=1$. Suppose a heat source, which does not change with time and set $h(x)=C^{-1} J(x)$.

Let us assume in addition $0<a<b$. It is proven in [12] that in this case Equation (21) is a generalized subdiffusion equation and admits the form (6) with kernel (see [12])

$$
k(t)=\frac{a}{b} \delta(t)+\left(1-\frac{a}{b}\right) \frac{1}{b} t^{\alpha-1} E_{\alpha, \alpha}\left(-\frac{1}{b} t^{\alpha}\right) ; \quad \widehat{k}(s)=\frac{1+a s^{\alpha}}{1+b s^{\alpha}} .
$$

By the use of (12) and (17) one finds that the corresponding Sonine kernel $l(t)$ obeys

$$
l(t)=1+\left(\frac{b}{a}-1\right) E_{\alpha}\left(-\frac{1}{a} t^{\alpha}\right) ; \quad \widehat{l}(s)=\frac{1+b s^{\alpha}}{s\left(1+a s^{\alpha}\right)} .
$$

For the source factor $q(t)$ we derive from (22) $q(t)=1+\left(\frac{a}{b}-1\right) E_{\alpha}\left(-\frac{1}{b} t^{\alpha}\right)$. Therefore, in this case $q(t)$ is a continuous, positive and increasing function with $q(0)=a / b>0$.

\section{Generalized Relaxation Equation}

This section contains preliminary results on the inhomogeneous generalized relaxation equation, for more details we refer to [35,36].

The solution of the inhomogeneous generalized relaxation equation

$$
\left(\mathbb{D}_{t}^{(k)} v\right)(t)+\lambda v(t)=r(t), \quad \lambda \geq 0, \quad t>0, \quad v(0)=a,
$$

admits the representation

$$
v(t)=a S(t ; \lambda)+\int_{0}^{t} G(t-\tau ; \lambda) r(\tau) d \tau
$$

where the functions $S(t ; \lambda)$ and $G(t ; \lambda)$ are defined in Laplace domain as follows

$$
\widehat{S}(s ; \lambda)=\frac{\widehat{k}(s)}{s \widehat{k}(s)+\lambda}, \quad \widehat{G}(s ; \lambda)=\frac{1}{s \widehat{k}(s)+\lambda}, \quad \lambda \geq 0 .
$$

In the particular case $\lambda=0$

$$
S(t ; 0) \equiv 1, \quad G(t ; 0)=l(t), \quad t \geq 0,
$$

where $l(t)$ is the corresponding Sonine kernel to the kernel $k(t)$, see (12).

Next, we summarize some properties of the functions $S(t ; \lambda)$ and $G(t ; \lambda)$, see e.g., [35], Theorem 2.2 and [36], Lemma 4.1, and [40], Theorem 5.2.

Proposition 1. Assume the kernel $k$ satisfies conditions (3) and $\lambda \geq 0$. Then Equation (24) has a unique solution given by (25), where the functions $S(t ; \lambda)$ and $G(t ; \lambda)$ are locally integrable and 
completely monotone with respect to $t \in \mathbb{R}_{+}$, and admit holomorphic extensions to the half-plane $\mathbb{C}_{+}$. Moreover,

$$
\frac{d}{d t} S(t ; \lambda)=-\lambda G(t ; \lambda), \quad t \geq 0
$$

and for any $\lambda \geq \lambda^{*}>0$

$$
S(t ; \lambda) \leq S\left(t ; \lambda^{*}\right), \quad t \geq 0
$$

and

$$
c \leq \lambda \int_{0}^{T} G(t ; \lambda) d t<1, T>0,
$$

where the constant $c=1-S\left(T ; \lambda^{*}\right)>0$ is independent of $\lambda$.

Let us note that for any $\lambda \geq 0$ and $t>0$

$$
\int_{0}^{t} G(\tau ; \lambda) d \tau>0
$$

For $\lambda>0$ this follows from (30). If $\lambda=0$ then (31) reduces to $(1 * l)(t)>0$, see (27). Since $l(t) \in \mathcal{C M F}$ then $(1 * l)(t) \in \mathcal{B F}$. This implies that it is positive for all $t>0$, as a continuous, non-negative and non-decreasing function for $t \geq 0$, which is analytic for $t>0$, see property (P4) in Section 2.

In the particular case of the relaxation equation with the Caputo fractional derivative of order $\alpha \in(0,1)$ the representation are valid $S(t ; \lambda)=E_{\alpha}\left(-\lambda t^{\alpha}\right)$ and $G(t ; \lambda)=$ $t^{\alpha-1} E_{\alpha, \alpha}\left(-\lambda t^{\alpha}\right)$, where $E_{\alpha, \beta}(\cdot)$ is the Mittag-Leffler function. In this case, (29) is the wellknown relation

$$
\frac{\mathrm{d}}{\mathrm{d} t} E_{\alpha}\left(-\lambda t^{\alpha}\right)=-\lambda t^{\alpha-1} E_{\alpha, \alpha}\left(-\lambda t^{\alpha}\right)
$$

and estimates (30) are used in [17,19-21] for the proof of uniqueness or/and (conditional) stability results of inverse problems for time-fractional diffusion equations.

Inequalities (30) and (31) are used in $[35,36]$ for the study of inverse source problems for generalized subdiffusion equations, based on eigenfunction expansion.

\section{Biorthonormal Pair of Riesz Bases}

Let us denote by $\langle.,$.$\rangle the inner product in L^{2}(0,1)$, i.e., $\langle f, g\rangle=\int_{0}^{1} f(x) g(x) \mathrm{d} x$. The norm $\|\cdot\|_{L^{2}(0,1)}$ in $L^{2}(0,1)$ is $\|f\|_{L^{2}(0,1)}=\langle f, f\rangle^{1 / 2}$. The Sobolev space $H^{2}(0,1)$ is defined as the subset of functions $f \in L^{2}(0,1)$, such that $f$ and its weak derivatives $f^{\prime}, f^{\prime \prime}$ have a finite $L^{2}$ norm. Consider the space $H^{2}(0,1)$ equipped with the norm $\|f\|_{H^{2}(0,1)}=$ $\|f\|_{L^{2}(0,1)}+\left\|f^{\prime \prime}\right\|_{L^{2}(0,1)}$.

We apply the technique of spectral decomposition with respect to generalized eigenfunctions for the non-selfadjoint operator defined by the boundary conditions (7), see e.g., [41-43]. Since the eigenvalues of the spectral problem for the second order differential operator with the boundary conditions (7) are $4 \pi^{2} n^{2}, n \in \mathbb{N}_{0}$, and for $n \neq 0$ each eigenvalue has multiplicity 2 , the system of eigenfunctions is not complete and must be supplemented with associated functions. In this way, the following system of generalized eigenfunctions (eigenfunctions and associated eigenfunctions) $\left\{X_{0, n}, X_{1, n}, X_{2, n}\right\}_{n=1}^{\infty}$ is obtained:

$$
X_{1,0}(x)=2(1-x), \quad X_{1, n}(x)=4(1-x) \cos \lambda_{n} x, \quad X_{2, n}(x)=4 \sin \lambda_{n} x, \quad \lambda_{n}=2 \pi n, n \in \mathbb{N} \text {. }
$$

This system of functions is a basis in $L^{2}(0,1)$. Any function $f \in L^{2}(0,1)$ admits the following unique formal spectral expansion

$$
f(x)=f_{1,0} X_{1,0}(x)+\sum_{n=1}^{\infty}\left\{f_{1, n} X_{1, n}(x)+f_{2, n} X_{2, n}(x)\right\}
$$


with coefficients defined by the identities

$$
f_{j, n}=\left\langle f, Y_{j, n}\right\rangle,
$$

where the system of functions $\left\{Y_{0, n}, Y_{1, n}, Y_{2, n}\right\}_{n=1}^{\infty}$ is

$$
Y_{1,0}(x)=1, Y_{1, n}(x)=\cos \lambda_{n} x, Y_{2, n}(x)=x \sin \lambda_{n} x, \lambda_{n}=2 \pi n, n \in \mathbb{N} .
$$

It is a basis in $L^{2}(0,1)$, orthonormal to $\left\{X_{0, n}, X_{1, n}, X_{2, n}\right\}_{n=1}^{\infty}$, that is $\left\langle X_{i, n}, Y_{j, m}\right\rangle=\delta_{i j} \delta_{n m}$.

The system of functions $\left\{X_{0, n}, X_{1, n}, X_{2, n}\right\}_{n=1}^{\infty}$ defined in (32) is a Riesz basis in $L^{2}(0,1)[41-43]$. This means that for any $f \in L^{2}(0,1)$ the following estimates are satisfied

$$
C^{-}\|f\|_{L^{2}(0,1)}^{2} \leq f_{1,0}^{2}+\sum_{n=1}^{\infty}\left\{f_{1, n}^{2}+f_{2, n}^{2}\right\} \leq C^{+}\|f\|_{L^{2}(0,1)}^{2}
$$

for some constants $C^{ \pm}>0$.

Proposition 2. Assume $f \in H^{2}(0,1)$ and $f(1)=0, f^{\prime}(0)=f^{\prime}(1)$. Then there exist constants $C_{1}^{ \pm}$, such that the estimates hold true

$$
C_{1}^{-}\|f\|_{H^{2}(0,1)}^{2} \leq f_{1,0}^{2}+\sum_{n=1}^{\infty}\left(1+\lambda_{n}^{4}\right)\left\{f_{1, n}^{2}+f_{2, n}^{2}\right\} \leq C_{1}^{+}\|f\|_{H^{2}(0,1)}^{2}
$$

Proof. Let $f$ satisfies the assumptions of the proposition. Taking into account the elementary inequalities $a^{2}+b^{2} \leq(a+b)^{2} \leq 2\left(a^{2}+b^{2}\right)$ for $a, b \geq 0$, it is sufficient to prove the estimates

$$
C_{2}^{-}\left\|f^{\prime \prime}\right\|_{L^{2}(0,1)}^{2} \leq \sum_{n=1}^{\infty} \lambda_{n}^{4}\left\{f_{1, n}^{2}+f_{2, n}^{2}\right\} \leq C_{2}^{+}\left\|f^{\prime \prime}\right\|_{L^{2}(0,1)}^{2} .
$$

Applying twice integration by parts and taking into account that $f(1)=0, f^{\prime}(0)=f^{\prime}(1)$, we find by the use of (34) the following expressions for the coefficients of $f^{\prime \prime}(x)$

$f_{1,0}^{\prime \prime}=\left\langle f^{\prime \prime}, Y_{1,0}\right\rangle=0, f_{1, n}^{\prime \prime}=\left\langle f^{\prime \prime}, Y_{1, n}\right\rangle=-\lambda_{n}^{2} f_{1, n}, f_{2, n}^{\prime \prime}=\left\langle f^{\prime \prime}, Y_{2, n}\right\rangle=2 \lambda_{n} f_{1, n}-\lambda_{n}^{2} f_{2, n}, \quad n \in \mathbb{N}$.

In this way, we obtain the expansion

$$
f^{\prime \prime}(x)=\sum_{n=1}^{\infty}\left\{-\lambda_{n}^{2} f_{1, n} X_{1, n}(x)+\left(2 \lambda_{n} f_{1, n}-\lambda_{n}^{2} f_{2, n}\right) X_{2, n}(x)\right\},
$$

which gives by applying the Riesz basis property (36) the estimates

$$
C^{-}\left\|f^{\prime \prime}\right\|_{L^{2}(0,1)}^{2} \leq \sum_{n=1}^{\infty}\left\{\lambda_{n}^{4} f_{1, n}^{2}+\left(2 \lambda_{n} f_{1, n}-\lambda_{n}^{2} f_{2, n}\right)^{2}\right\} \leq C^{+}\left\|f^{\prime \prime}\right\|_{L^{2}(0,1)}^{2} .
$$

The lower bound in (38) follows easily from the lower bound in (40) using the inequalities $\left(2 \lambda_{n} f_{1, n}-\lambda_{n}^{2} f_{2, n}\right)^{2} \leq 8 \lambda_{n}^{2} f_{1, n}^{2}+2 \lambda_{n}^{4} f_{2, n}^{2}$ and $\lambda_{n}^{2}<\lambda_{n}^{4}, n \in \mathbb{N}$.

To deduce the upper bound in (38) from the upper bound in (40), we use the following identities obtained from (34) by integration by parts

$$
\begin{aligned}
& f_{1, n}=\left\langle f, \cos \lambda_{n} x\right\rangle=-\frac{1}{\lambda_{n}^{2}}\left\langle f^{\prime \prime}, \cos \lambda_{n} x\right\rangle ; \\
& f_{2, n}=\left\langle x f(x), \sin \lambda_{n} x\right\rangle=-\frac{1}{\lambda_{n}^{2}}\left\langle x f^{\prime \prime}(x), \sin \lambda_{n} x\right\rangle-\frac{2}{\lambda_{n}^{3}}\left\langle f^{\prime \prime}(x), \cos \lambda_{n} x\right\rangle .
\end{aligned}
$$


By applying the Bessel inequality for the trigonometric series identity (41) yields

$$
\sum_{n=1}^{\infty} \lambda_{n}^{4} f_{1, n}^{2}=\sum_{n=1}^{\infty}\left\langle f^{\prime \prime}, \cos \lambda_{n} x\right\rangle^{2} \leq\left\|f^{\prime \prime}\right\|_{L^{2}(0,1)}^{2}
$$

and, in a similar way, (42) implies

$$
\begin{aligned}
\sum_{n=1}^{\infty} \lambda_{n}^{4} f_{2, n}^{2} & \leq C_{1} \sum_{n=1}^{\infty}\left\langle x f^{\prime \prime}(x), \sin \lambda_{n} x\right\rangle^{2}+C_{2} \sum_{n=1}^{\infty}\left\langle f^{\prime \prime}(x), \cos \lambda_{n} x\right\rangle^{2} \\
& \leq C_{1}\left\|x f^{\prime \prime}(x)\right\|_{L^{2}(0,1)}^{2}+C_{2}\left\|f^{\prime \prime}(x)\right\|_{L^{2}(0,1)}^{2} \\
& \leq C_{3}\left\|f^{\prime \prime}\right\|_{L^{2}(0,1)}^{2} .
\end{aligned}
$$

In this way, combining (43) and (44), the upper bound in (38) is also established.

\section{Formal Spectral Expansions for the Solution}

In this section, we find formal spectral expansions for the unknown functions $h(x)$ and $u(x, t)$ in the inverse source problem (6)-(8). Suppose

$$
\begin{aligned}
h(x) & =h_{1,0} X_{1,0}(x)+\sum_{n=1}^{\infty}\left\{h_{1, n} X_{1, n}(x)+h_{2, n} X_{2, n}(x)\right\} \\
u(x, t) & =u_{1,0}(t) X_{1,0}(x)+\sum_{n=1}^{\infty}\left\{u_{1, n}(t) X_{1, n}(x)+u_{2, n}(t) X_{2, n}(x)\right\} .
\end{aligned}
$$

To find the unknown coefficients in (45) and (46), we insert these expansions in Equation (6) and, by taking into account (39) and the initial condition $u(x, 0)=0$, we obtain by the uniqueness property of the spectral expansion the following system of equations

$$
\begin{aligned}
& \mathbb{D}_{t}^{(k)} u_{1, n}(t)+\lambda_{n}^{2} u_{1, n}(t)=h_{1, n} q(t), \quad u_{1, n}(0)=0, \quad n \in \mathbb{N}_{0}, \\
& \mathbb{D}_{t}^{(k)} u_{2, n}(t)+\lambda_{n}^{2} u_{2, n}(t)=2 \lambda_{n} u_{1, n}(t)+h_{2, n} q(t), u_{2, n}(0)=0, \quad n \in \mathbb{N} .
\end{aligned}
$$

We solve first relaxation equation (47) and after that (48). In this way, by applying formula (25) we obtain

$$
\begin{aligned}
& u_{1, n}(t)=h_{1, n} A_{n}(t), \quad n \in \mathbb{N}_{0}, \\
& u_{2, n}(t)=h_{2, n} A_{n}(t)+2 \lambda_{n} h_{1, n} B_{n}(t), \quad n \in \mathbb{N} .
\end{aligned}
$$

Here the functions $A_{n}(t)$ and $B_{n}(t)$ are defined as follows

$$
A_{n}(t)=\left(q * G_{n}\right)(t), \quad B_{n}(t)=\left(q * G_{n} * G_{n}\right)(t) ; \quad G_{n}(t)=G\left(t ; \lambda_{n}^{2}\right),
$$

where $G(t ; \lambda)$ is defined in (26). In this way, we obtain

$$
u(x, t)=h_{1,0} A_{0}(t) X_{1,0}(x)+\sum_{n=1}^{\infty}\left\{h_{1, n} A_{n}(t) X_{1, n}(x)+\left(h_{2, n} A_{n}(t)+2 \lambda_{n} h_{1, n} B_{n}(t)\right) X_{2, n}(x)\right\} .
$$

Therefore, the function $g(x)=u(x, T)$ admits the spectral expansion

$$
g(x)=h_{1,0} A_{0}(T) X_{1,0}(x)+\sum_{n=1}^{\infty}\left\{h_{1, n} A_{n}(T) X_{1, n}(x)+\left(h_{2, n} A_{n}(T)+2 \lambda_{n} h_{1, n} B_{n}(T)\right) X_{2, n}(x)\right\} .
$$

By the uniqueness property of the spectral expansion, from (53) we deduce

$$
g_{1, n}=h_{1, n} A_{n}(T), n \in \mathbb{N}_{0} ; \quad g_{2, n}=h_{2, n} A_{n}(T)+2 \lambda_{n} h_{1, n} B_{n}(T), n \in \mathbb{N} .
$$


Since $A_{n}(T)>0$ for all $n \in \mathbb{N}_{0}$ (see Proposition 3), it follows from (54)

$$
h_{1, n}=\frac{1}{A_{n}(T)} g_{1, n}, n \in \mathbb{N}_{0} ; \quad h_{2, n}=\frac{1}{A_{n}(T)}\left(g_{2, n}-2 \lambda_{n} g_{1, n} \frac{B_{n}(T)}{A_{n}(T)}\right), n \in \mathbb{N} .
$$

Relations (54) and (55) define a mapping between the overdetermination function $g(x)$ and the source factor $h(x)$ and our main goal is to determine the properties of this mapping.

Plugging the coefficients $h_{j, n}$ in (52), we derive the coefficients $u_{j, n}(t)$ in the spectral expansion (46) of the solution $u(x, t)$

$$
\begin{aligned}
& u_{1, n}(t)=g_{1, n} \frac{A_{n}(t)}{A_{n}(T)}, n \in \mathbb{N}_{0} \\
& u_{2, n}(t)=g_{2, n} \frac{A_{n}(t)}{A_{n}(T)}+2 \lambda_{n} g_{1, n}\left(\frac{B_{n}(t)}{A_{n}(T)}-\frac{A_{n}(t) B_{n}(T)}{A_{n}^{2}(T)}\right), n \in \mathbb{N} .
\end{aligned}
$$

In this way, inserting (55) in (45), and (56), (57) in (46), we obtain the formal expansions for $h(x)$ and $u(x, t)$. The functions $A_{n}(t)$ and $B_{n}(t)$ in these expansions depend on the specific memory kernel $k(t)$ via $G_{n}(t)$ and the time-dependent source factor $q(t)$.

\section{Uniqueness of Solution and Stability Estimates in Sobolev Spaces}

In this section, we prove that under some assumptions on the overdetermination function $g(x)$ and the time-dependent source factor $q(t)$, the formal expansions (45) with coefficients (55) and (46) with coefficients (56) and (57) define a unique solution $(h, u)$ to the considered inverse source problem.

For the given time-dependent source factor $q(t)$ we assume

$$
q \in C[0, T] ; \quad q(t) \geq 0, t \in[0, T] ; \quad q(T)>0 .
$$

Let us note that since $q(t)$ is a continuous function for $t \in(0, T]$, the assumption $q(T)>0$ implies that there exist $q_{0}>0$ and $T_{1} \in(0, T)$, such that

$$
q(t) \geq q_{0}>0 \text { for all } t \in\left[T_{1}, T\right] .
$$

First, we prove some estimates for the functions $A_{n}(t)$ and $B_{n}(t)$.

Proposition 3. Let $T>0$ be arbitrarily fixed. Assume that conditions (3) and (58) for the functions $k(t)$ and $q(t)$, respectively, are satisfied. Then the functions $A_{0}(t), A_{n}(t), B_{n}(t), n \in \mathbb{N}$, are continuous and non-negative on $[0, T]$, vanish at $t=0$, and $A_{n}(T)>0$ for all $n \in \mathbb{N}_{0}$. Moreover, the following estimates for $t \in[0, T]$ and $n \in \mathbb{N}$ are satisfied:

$$
\begin{aligned}
& A_{n}(T) \geq C_{1} / \lambda_{n}^{2}, \\
& A_{n}(t) \leq C_{2} / \lambda_{n}^{2}, \\
& B_{n}(t) \leq C_{2} / \lambda_{n}^{4},
\end{aligned}
$$

where the constants $C_{j}>0, j=1,2$, are independent of $n$.

Proof. The functions $A_{n}(t)$ and $B_{n}(t)$ are continuous and non-negative as convolutions of functions with these properties. Fix $T_{1} \in(0, T)$, such that (59) is satisfied. For $n \geq 0$ we deduce

$$
A_{n}(T)=\int_{0}^{T} q(t) G_{n}(T-t) \mathrm{d} t \geq \int_{T_{1}}^{T} q(t) G_{n}(T-t) \mathrm{d} t \geq q_{0} \int_{0}^{T-T_{1}} G_{n}(t) \mathrm{d} t,
$$

due to the non-negativity of the functions under the integral sign and inequality (59). Then (63) together with (31) implies $A_{n}(T)>0, n \in \mathbb{N}_{0}$. 
Estimates (63) and (30) in Proposition 1 imply for $n \in \mathbb{N}$

$$
\lambda_{n}^{2} A_{n}(T) \geq q_{0}\left(1-S\left(T-T_{1} ; \lambda_{1}^{2}\right)\right)>0,
$$

where $\lambda_{1}^{2}=4 \pi^{2}$ is the smallest positive eigenvalue and the function $S(t ; \lambda)$ is defined in (26). In this way, (60) is established with $C_{1}=q_{0}\left(1-S\left(T-T_{1} ; \lambda_{1}^{2}\right)\right)>0$.

Next, we prove estimates (61) and (62). The representation in (51) and the estimate from above in (30) yield

$$
A_{n}(t)=\left(q * G_{n}\right)(t) \leq\|q\|_{C[0, T]}\left(1 * G_{n}\right)(t) \leq \frac{C_{2}}{\lambda_{n}^{2}}
$$

which implies (61) with $C_{2}=\|q\|_{C[0, T]}>0$. By applying (61) and (30) we derive (62):

$$
B_{n}(t)=\left(q * G_{n} * G_{n}\right)(t)=\left(A_{n} * G_{n}\right)(t) \leq \frac{C_{2}}{\lambda_{n}^{2}}\left(1 * G_{n}\right)(t) \leq \frac{C_{2}}{\lambda_{n}^{4}} .
$$

Since $\left(1 * G_{n}\right)(0)=0$, the above two estimates also imply that $A_{n}(0)=0$ and $B_{n}(0)=0$.

We are ready to formulate and prove the main result of this work.

Theorem 1. Let $T>0$ be arbitrarily fixed. For any given $g \in H^{2}(0,1)$, such that $g(1)=0$, $g^{\prime}(0)=g^{\prime}(1)$, there exists a unique solution $(h, u)$ to problem (6)-(8), satisfying $h \in L^{2}(0,1)$ and $u \in C\left([0, T] ; H^{2}(0,1)\right)$. The functions $h(x)$ and $u(x, t)$ are defined by the spectral expansions (45) and (46) with coefficients given in (55), (56) and (57). Moreover, there exist constants $\underline{c}>0$ and $\bar{c}>0$, such that

$$
\underline{c}\|g\|_{H^{2}(0,1)} \leq\|h\|_{L^{2}(0,1)} \leq \bar{c}\|g\|_{H^{2}(0,1)} .
$$

Proof. Uniqueness of the solution follows from the uniqueness property of the spectral expansions and the fact that $A_{n}(T) \neq 0$, for all $n \geq 0$ (see Proposition 3). Indeed, if $g \equiv 0$, then $g_{j, n}=0$, and therefore, (55)-(57) imply $h_{j, n}=0, u_{j, n}=0$, i.e., all coefficients in the expansions (45) and (46) vanish and, therefore, $h \equiv 0$ and $u \equiv 0$.

The initial condition $u(x, 0)=0$ is satisfied since $A_{n}(0)=0$ and $B_{n}(0)=0$. The two boundary conditions (7) hold by construction, since they are satisfied by the basis functions $X_{1, n}(x)$ and $X_{2, n}(x)$ defined in (32).

Applying estimates (60), (62), and taking into account the inequalities $(a \pm b)^{2} \leq$ $2\left(a^{2}+b^{2}\right)$ and $\lambda_{n}>1, n \in \mathbb{N}$, identities (55) imply

$$
h_{1, n}^{2} \leq C_{1} \lambda_{n}^{4} g_{1, n}^{2}, \quad h_{2, n}^{2} \leq C_{2} \lambda_{n}^{4}\left(g_{1, n}^{2}+g_{2, n}^{2}\right), \quad n \in \mathbb{N} .
$$

Estimates (65) together the lower bound in (36) with $f=h$ and the upper bound in (37) with $f=g$ imply the upper bound in (64). By applying similar argument, we obtain from (54) by the use of Proposition 3 the estimates

$$
\lambda_{n g_{1, n}^{4}}^{4} \leq C_{3} h_{1, n}^{2}, \quad \lambda_{n}^{4} g_{2, n}^{2} \leq C_{4}\left(h_{1, n}^{2}+h_{2, n}^{2}\right), \quad n \in \mathbb{N} .
$$

The last estimates together with the lower bound in (37) with $f=g$ and the upper bound in (36) with $f=h$ yield the lower bound in (64).

Furthermore, Proposition 3 implies for $t \in[0, T]$

$$
\left(\frac{A_{n}(t)}{A_{n}(T)}\right)^{2} \leq C_{5},\left(\frac{B_{n}(t)}{A_{n}(T)}-\frac{A_{n}(t) B_{n}(T)}{A_{n}^{2}(T)}\right)^{2} \leq 2\left(\frac{B_{n}(t)}{A_{n}(T)}\right)^{2}+2 C_{5}\left(\frac{B_{n}(T)}{A_{n}(T)}\right)^{2} \leq \frac{C_{6}}{\lambda_{n}^{2}} .
$$


Representations (56) and (57) for the coefficients of the solution $u(x, t)$ yield by the use of estimates (67)

$$
u_{1, n}^{2}(t) \leq C_{7} g_{1, n}^{2}, \quad u_{2, n}^{2}(t) \leq C_{8}\left(g_{1, n}^{2}+g_{2, n}^{2}\right), \quad t \in[0, T], n \in \mathbb{N} .
$$

Since $g \in H^{2}(0,1)$, it follows from (68) that

$$
\|u\|_{C\left([0, T] ; H^{2}(0,1)\right)} \leq C_{9}\|g\|_{H^{2}(0,1)}<\infty .
$$

Therefore $u \in C\left([0, T] ; H^{2}(0,1)\right)$. The proof of the theorem is complete.

It is worth noting that if $q(t) \in C^{1}[0, T]$ then the inverse source problem under consideration can be reduced to a Fredholm equation of second kind, by applying the argument proposed in [17]. In general, this fact is well known for parabolic equations (e.g., [44]). In this case, it is sufficient to prove the uniqueness for the inverse problem, and then the stability follows by the Fredholm alternative. However, in the present work, continuous differentiability of $q(t)$ is not assumed, see (58), and a specialized argument is needed.

Adopting the method of [17], the more general case $q=q(x, t)$ can be also treated by the use of the generalized eigenfunction expansion, provided the function $q(x, t)$ obeys sufficient regularity. Although no explicit series representation of the source term $h(x)$ can be derived in this case, the estimates (64) can be established by applying the Fredholm alternative in $L^{2}(0,1)$.

We close this section with a conditional stability result. It concerns the case when the overdetermination function $g$ is only square integrable, $g \in L^{2}(0,1)$, which is relevant in the study of stability with respect to the noise level applied to the input, see (9). In this case, an a priori bound assumption for the unknown function $h(x)$ is needed, see e.g., [21]. Let us assume the following a priori bound: $\|h\|_{H^{2}(0,1)} \leq E$. Then

$$
\|h\|_{L^{2}(0,1)} \leq C_{E}\|g\|_{L^{2}(0,1)^{\prime}}^{1 / 2}
$$

where the constant $C_{E}$ depends on $E$ and the constants appearing in the inequalities (36), (37), (65) and (66). Estimate (69) can be derived adapting the technique in [35] to the case of generalized eigenfunction expansions by the use of the estimates (36), (37), (65) and (66) and the Cauchy-Schwarz inequality. Details of the proof are omitted here.

\section{Concluding Remarks}

This work is concerned with an inverse source problem for the one-dimensional generalized subdiffusion equation on a bounded interval. The differences to the classical case are as follows. First, temporal derivative in the diffusion equation is replaced by a general convolutional derivative in time. This allows the modeling of memory effects of the described physical process. Second, traditional Dirichlet boundary condition is applied at the one side of the interval only and the other boundary condition is nonlocal.

The main result of the paper is Theorem 1 establishing a two-sided estimate of the $L^{2}$ norm of the spatial part $h(x)$ of the forcing term via the Sobolev $H^{2}$ norm of the final overdetermination function $g(x)$. The derived estimates extend earlier obtained results on the classical diffusion problem and the anomalous diffusion problem with the Caputo time-fractional derivative subject to Dirichlet boundary conditions. The proofs are based on generalized eigenfunction expansions with respect to a biorthogonal pair of bases, together with estimates of the special functions in time appearing in the expansions, which inherit relevant properties of Mittag-Leffler functions.

It is feasible to extend the results to multidimensional cases, such as the two-dimensional nonlocal problem in [34].

Another approach to further study of such types of inverse problems is to employ the general theory for parabolic equations [44]. Under appropriate assumptions on the given 
functions the problem can be reduced to a Fredholm equation of the second kind and then the Fredholm alternative can be applied.

Funding: This work is supported by the National Scientific Program "Information and Communication Technologies for a Single Digital Market in Science, Education and Security (ICTinSES)", contract No DO1-205/23.11.2018, financed by the Ministry of Education and Science in Bulgaria.

Institutional Review Board Statement: Not applicable.

Informed Consent Statement: Not applicable.

Data Availability Statement: Not applicable.

Acknowledgments: The author is grateful to the anonymous reviewers for the constructive comments. The work is performed in the frames of the bilateral research project "Operators, differential equations and special functions of Fractional Calculus-numerics and applications" between Bulgarian Academy of Sciences and Serbian Academy of Sciences and Arts.

Conflicts of Interest: The author declares no conflict of interest.

\section{References}

1. Metzler, R.; Jeon, J.H.; Cherstvy, A.G.; Barkai, E. Anomalous diffusion models and their properties: Non-stationarity, nonergodicity, and ageing at the centenary of single particle tracking. Phys. Chem. Chem. Phys. 2014, 16, 24128. [CrossRef]

2. Kochubei, A.; Luchko, Y. (Eds.) Fractional Differential Equations; De Gruyter: Berlin, Germany; Boston, MA, USA, 2019.

3. Guo, B.; Pu, X.; Huang, F. Fractional Partial Differential Equations and Their Numerical Solutions; World Scientific: Singapore, 2015.

4. Sandev, T.; Sokolov, I.M.; Metzler, R.; Chechkin, A. Beyond monofractional kinetics. Chaos Solitons Fractals 2017, $102,210-217$. [CrossRef]

5. Sandev, T.; Metzler, R.; Chechkin, A. From continuous time random walks to the generalized diffusion equation. Fract. Calc. Appl. Anal. 2018, 21, 10-28. [CrossRef]

6. Sandev, T.; Tomovski, Z.; Dubbeldam, J.L.A.; Chechkin, A. Generalized diffusion-wave equation with memory kernel. J. Phys. Math. Theor. 2019, 52, 015201. [CrossRef]

7. Kochubei, A. General fractional calculus, evolution equations, and renewal processes. Integr. Equ. Oper. Theory 2011, 71, 583-600. [CrossRef]

8. Luchko, Y.; Yamamoto, M. The general fractional derivative and related fractional differential equations. Mathematics 2020, 8, 2115. [CrossRef]

9. Luchko, Y. Operational calculus for the general fractional derivative and its applications. Fract. Calc. Appl. Anal. 2021, 24, 338-375. [CrossRef]

10. Schumer, R.; Benson, D.; Meerschaert, M.; Baeumer, B. Fractal mobile/immobile solute transport. Water Resour. Res. 2003, 39, SBH131-SBH1312. [CrossRef]

11. Atanacković, T.M.; Pilipović, S.; Stanković, B.; Zorica, D. Fractional Calculus with Applications in Mechanics: Vibrations and Diffusion Processes; John Wiley \& Sons: London, UK, 2014.

12. Bazhlekova, E.; Bazhlekov, I. Transition from diffusion to wave propagation in fractional Jeffreys-type heat conduction equation. Fractal Fract. 2020, 4, 32. [CrossRef]

13. Schilling, R.; Song, R.; Vondraček, Z. Bernstein Functions: Theory and Applications; De Gruyter: Berlin, Germany, 2010.

14. Clement, P.; Prüss, J. Completely positive measures and Feller semigroups. Math. Ann. 1990, 287, 73-105. [CrossRef]

15. Prüss, J. Evolutionary Integral Equations and Applications; Birkhäuser: Basel, Switzerland, 1993.

16. Vergara, V.; Zacher, R. Optimal Decay Estimates for Time-Fractional and Other NonLocal Subdiffusion Equations via Energy Methods. SIAM J. Math. Anal. 2015, 47, 210-239. [CrossRef]

17. Sakamoto, K.; Yamamoto, M. Inverse source problem with a final overdetermination for a fractional diffusion equation. Math. Control Relat. Fields 2011, 1, 509-518. [CrossRef]

18. Tuan, N.H.; Debbouche, A.; Ngoc, T.B. Existence and regularity of final value problems for time fractional wave equations. Comput. Math. Appl. 2019, 78, 1396-1414. [CrossRef]

19. Wei, T.; Wang, J. A modified quasi-boundary value method for an inverse source problem of the time-fractional diffusion equation. Appl. Num. Math. 2014, 78, 95-111. [CrossRef]

20. Zhang, Y.; Xu, X. Inverse source problem for a fractional diffusion equation. Inverse Probl. 2011, 27, 035010. [CrossRef]

21. Zhang, Z.; Wei, T. Identifying an unknown source in time-fractional diffusion equation by a truncation method. Appl. Math. Comp. 2013, 219, 5972-5983. [CrossRef]

22. Jin, B.; Rundell, W. A tutorial on inverse problems for anomalous diffusion processes. Inverse Probl. 2015, 31, 035003. [CrossRef]

23. Tuan, N.; Kirane, M.; Hoan, L.; Long, L. Identification and regularization for unknown source for a time-fractional diffusion equation. Comput. Math. Appl. 2017, 73, 931-950. [CrossRef] 
24. Slodička, M.; Šišková, K.; Bockstal, K.V. Uniqueness for an inverse source problem of determining a space dependent source in a time-fractional diffusion equation. Appl. Math. Lett. 2019, 91, 15-21. [CrossRef]

25. Slodička, M. Uniqueness for an inverse source problem of determining a space-dependent source in a non-autonomous time-fractional diffusion equation. Fract. Calc. Appl. Anal. 2020, 23, 1702-1711. [CrossRef]

26. Kinash, N.; Janno, J. An inverse problem for a generalized fractional derivative with an application in reconstruction of time- and space-dependent sources in fractional diffusion and wave equations. Mathematics 2019, 7, 1138. [CrossRef]

27. Kinash, N.; Janno, J. Inverse problems for a generalized subdiffusion equation with final overdetermination. Math. Model. Anal. 2019, 24, 236-262. [CrossRef]

28. Kinash, N.; Janno, J. Inverse problems for a perturbed time fractional diffusion equation with final overdetermination. Math. Meth. Appl. Sci. 2018, 41, 1925-1943. [CrossRef]

29. Kaliev, I.; Sabitova, M. Problems of determining the temperature and density of heat sources from the initial and final temperatures. J. Appl. Indust. Math. 2010, 4, 332-339. [CrossRef]

30. Kirane, M.; Malik, S. Determination of an unknown source term and the temperature distribution for the linear heat equation involving fractional derivative in time. Appl. Math. Comp. 2011, 218, 163-170. [CrossRef]

31. Furati, K.; Iyiola, O.; Kirane, M. An inverse problem for a generalized fractional diffusion. Appl. Math. Comp. 2014, 249 , 24-31. [CrossRef]

32. Furati, K.; Iyiola, O.; Mustapha, K. An inverse source problem for a two-parameter anomalous diffusion with local time datum. Comput. Math. Appl. 2017, 73, 1008-1015. [CrossRef]

33. Ali, M.; Aziz, S.; Malik, S.A. Inverse problem for a multi-term fractional differential equation. Fract. Calc. Appl. Anal. 2020, 23, 799-821. [CrossRef]

34. Kirane, M.; Malik, S.; Al-Gwaiz, M. An inverse source problem for a two dimensional time fractional diffusion equation with nonlocal boundary conditions. Math. Meth. Appl. Sci. 2013, 36, 1056-1069. [CrossRef]

35. Bazhlekova, E. Estimates for a general fractional relaxation equation and application to an inverse source problem. Math. Met. Appl. Sci. 2018, 41, 9018-9026. [CrossRef]

36. Bazhlekova, E.; Bazhlekov, I. Identification of a space-dependent source term in a nonlocal problem for the general time-fractional diffusion equation. J. Comput. Appl. Math. 2021, 386, 113213. [CrossRef]

37. Gripenberg, G.; Londen, S.O.; Staffans, O. Volterra Integral and Functional Equations; Cambridge University Press: Cambridge, UK, 1990.

38. Bazhlekov, I.; Bazhlekova, E. Fractional derivative modeling of bioreaction-diffusion processes. AIP Conf. Proc. 2021, 2333,060006 [CrossRef]

39. Kilbas, A.A.; Srivastava, H.M.; Trujillo, J.J. Theory and Applications of Fractional Differential Equations; North-Holland Mathematics Studies; Elsevier: Amsterdam, The Netherlands, 2006.

40. Bazhlekova, E. Completely monotone multinomial Mittag-Leffler type functions and diffusion equations with multiple timederivatives. Fract. Calc. Appl. Anal. 2021, 24, 88-111. [CrossRef]

41. Ionkin, N. Solution of a boundary problem for a heat conduction equation with nonclassical boundary condition. Differ. Equ. 1977, 13, 294-304.

42. Ionkin, N.; Moiseev, E. A two-point boundary value problem for a heat conduction equation. Differ. Equ. 1979, 15, 1284-1295.

43. Moiseev, E. The solution of a nonlocal boundary value problem by the spectral method. Differ. Equ. 1999, 35, 1105-1112.

44. Prilepko, A.I.; Orlovsky, D.G.; Vasin, I.A. Methods for Solving Inverse Problems in Mathematical Physics; Marcel-Dekker: New York, NY, USA, 2000. 